\title{
Delirium after hip hemiarthroplasty for proximal femoral fractures in elderly patients: risk factors and clinical outcomes
}

This article was published in the following Dove Medical Press journal:

Clinical Interventions in Aging

\section{Louis de Jong' \\ Veronique AJIM van \\ Rijckevorsel' \\ Jelle W Raats' \\ Taco MAL Klem² \\ Tjallingius M Kuijper ${ }^{3}$ \\ Gert R Roukema'}

'Surgery Department, Maasstad Hospital, 3079 DZ Rotterdam, the Netherlands; ${ }^{2}$ Surgery Department, Franciscus Hospital, 3045 PM

Rotterdam, the Netherlands; ${ }^{3}$ Science Board, Maasstad Hospital, 3079 DZ

Rotterdam, the Netherlands
Correspondence: Louis de Jong Surgery Department, Maasstad Hospital, Maasstadweg 21, 3079 DZ Rotterdam, the Netherlands

Tel +3| 062392 I889

Email l.jong2@franciscus.nl
Background: The primary aim of the present study was to verify the potential risk factors for developing a delirium after hip fracture surgery. The secondary aim of this study was to examine the related clinical outcomes after a delirium developed post-hip fracture surgery.

Patients and methods: Data were extracted from a prospective hip fracture database and completed by retrospective review of the hospital records. A total of 463 patients undergoing hip fracture (hip hemiarthroplasty) surgery in a level II trauma teaching hospital between January 2011 and May 2016 were included. Delirium was measured using the Delirium Observation Screening Scale, the confusion assessment method, and an observatory judgment by geriatric medicine specialists

Results: The results showed that $26 \%$ of the patients $(n=121)$ developed a delirium during hospital stay with a median duration during admission of 5 days (IQR 3-7). The multivariable model showed that the development of delirium was significantly explained by dementia (OR 2.75, $P=0.001$ ), age (OR 1.06, $P=0.005$ ), and an infection during admission (pneumonia, deep surgical site infection, or urinary tract infection) (OR 1.23, $P=0.046$ ). After 1 year of follow-up, patients who developed delirium after hip fracture surgery were significantly more discharged to (semi-independent) nursing homes $(P<0.001)$ and had a significantly higher mortality rate $(P<0.001)$ compared to patients without delirium after hip fracture surgery.

Conclusions: The results showed that $26 \%$ of the patients undergoing hip fracture surgery developed a delirium. The risk factors including age, dementia, and infection during admission significantly predicted the development of the delirium. No association was confirmed between delirium and time of admission or time to surgery. The development of delirium after hip fracture surgery was subsequently found to be a significant predictor of admission to a nursing home and mortality after 1 year.

Keywords: delirium, hip fracture, femoral neck fracture, mortality, risk and prognostic factors, hemi athroplasty, fragile

\section{Introduction}

Due to the increasing life expectancy, the number of persons aged over 70 years will double within the next three decades. ${ }^{1}$ Especially, this increasing elderly population is at risk of hip fractures. ${ }^{2}$ Following a hip fracture, even frail patients with major comorbidities are in need for surgery, aiming for pain relief and early mobilization. ${ }^{3,4}$ In the frail population of hip fracture patients, complications after surgery are inevitable and high incidence rates of pneumonia (5.9\%), surgical site infections (5\%), and myocardial infarction (1.9\%) have been reported. ${ }^{3,5}$ The most frequent complication during admission after hip fracture surgery is delirium with incidence rates ranging between $23 \%$ and $39 \%$. $^{6-8}$ Postoperative delirium is subsequently associated with higher rates of impaired (c) hereby accept the Terms. Non-commercial uses of the work are permitted without any further permission from Dove Medical Press Limited, provided the work is properly attributed. For permission for commercial use of this work, please see paragraphs 4.2 and 5 of our Terms (https://www.dovepress.com/terms.php). 
functional and cognitive recovery, and a prolonged hospital stay with higher mortality rates and treatment costs. ${ }^{6,7,9-12}$ Reducing the rate of delirium after hip fracture surgery is important, and therefore multiple studies have focused on identifying risk factors for delirium or interventions. ${ }^{13-15}$ Risk factors that have been found in previous literature were age, cognitive impairment, type of admission (acute or elective), institutionalization, functional impairment, body mass index (BMI), level of albumin, American Society of Anesthesiologists (ASA) classification, general anesthesia, parkinsonism, polypharmacy, and vision impairment. ${ }^{13,16-18}$ Determination of risk factors for delirium after hip fracture surgery supports clinicians to identify patients at risk and enabling preventive measures. The aim of the present study was therefore to validate earlier suggested risk factors, which helps clinicians to identify high-risk patients for delirium. Moreover, the current study aimed to examine the delirium outcomes as adverse events, length of hospital stay, and mortality rates to gain a more complete insight into the outcomes of delirium after hip fracture surgery.

\section{Patients and methods}

\section{Patient selection}

This study enrolled patients who underwent surgical treatment for proximal hip fractures at a single-center level II trauma teaching hospital in Rotterdam (the Netherlands) from January 1, 2011 to May 1, 2016. All patients treated with a hemiarthroplasty (HA) were selected for analyses $(n=463)$ (Figure 1). The baseline characteristics and clinical outcomes were retrospectively obtained from the hospital records using a prospective registry. The study protocol was approved by the local ethics committee (L2017044, TWOR, Rotterdam). Because of the high percentage of cognitive dysfunction and no change in standard practice of care, the local ethics committee decided that patients' consent to review their medical records was not required. Patient data were stored anonymously and all the protocols were conducted in compliance with the Declaration of Helsinki. Moreover, no external funding was used for this study. All operations were performed by six orthopedic trauma surgeons in a laminar air flow theater, and the standard implant was a cemented unipolar (Palamed G, gentamicin impregnated cement; Heraeus, Hanau, Germany) prosthesis (Mathys CCA; Mathys Ltd. Bettlach, Switzerland). Standard antibiotic prophylaxis prior to surgery included 1-3 g cefazolin (Kefzol). Till January 2016, patients were hospitalized at the orthopedic department during admission. After January 2016, the patients were admitted at a geriatric trauma unit within the orthopedic department.

\section{Risk factors for delirium}

To evaluate the risk factors for delirium after hip fracture surgery, baseline characteristics of all the included patients, including age, gender, and medication, were collected. Comorbidities were divided into the following categories: rheumatoid arthritis, liver and kidney function, Parkinson's disease, cardio- or cerebrovascular disease, previous hip fracture surgery, oncological status, and the use of walking aids. Data on nutritional status were collected using the BMI and Short Nutritional Assessment Questionnaire for Residential Care score. Also, information about the living situation (at home or in a nursing home) prior to hospital admission was collected. In this study, patients living in a nursing home were care-dependent due to an extensive degree of either cognitive or physical impairment.

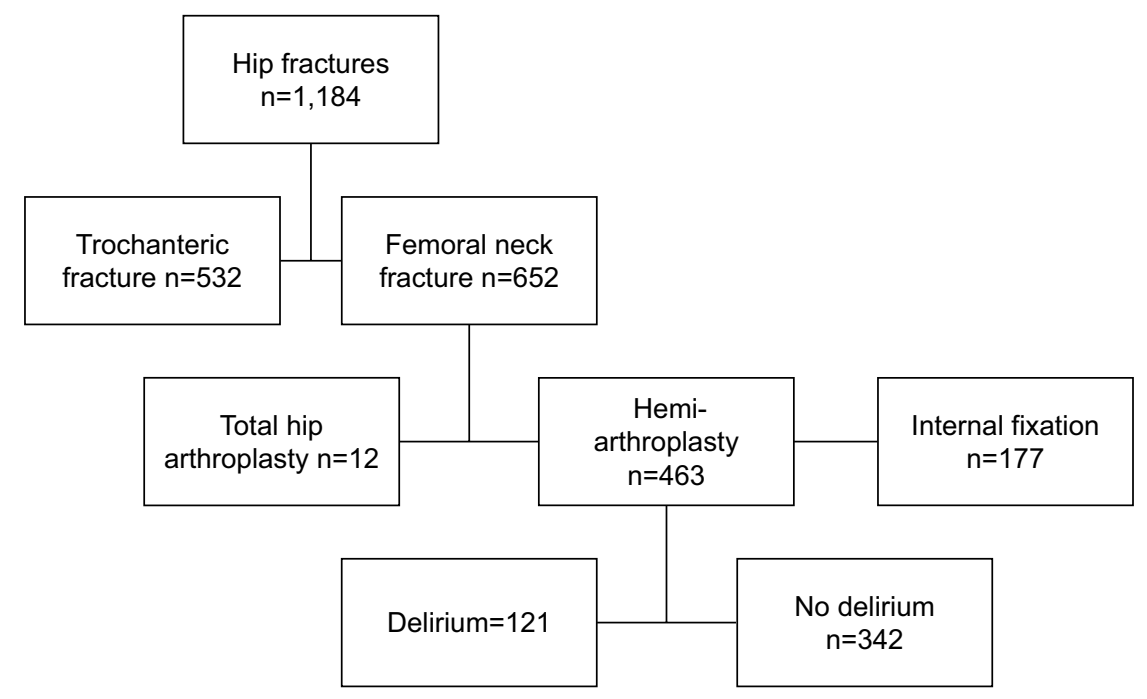

Figure I Flow chart of included patients. 


\section{Perioperative risk factors}

Since the primary objective of the current study was to examine perioperative risk factors for delirium after hip fracture surgery, we selected perioperative variables described previously in literature as risk factors for the development of delirium after HA. ${ }^{13,16-18}$ Perioperative factors were time to surgery (hours between admission and surgery), time of operation (surgery during day [hours]), moment of the week (during the week or weekend), estimated blood loss, and reoperations. Duration and type of anesthesia and the ASA score were collected. Furthermore, we used the Nottingham hip fracture score (NHFS) as a frailty prediction score for delirium, which combines the patient's comorbidities and general status. The NHFS is developed and validated for 30-day mortality rate prediction after hip fracture surgery, including age, sex, number of comorbidities, Mini-Mental Test Score Examination $\leq 6$ out of 10 , hemoglobin concentration on admission, residence, and the presence of a malignant disease in the past 20 years. ${ }^{19,20}$

\section{Delirium}

Patients were screened for delirium using the Delirium Observation Screening Scale (DOSS) scored by trained nurses during regular care. Three or more points on the DOSS were considered as highly indicative of delirium. All patients were visited on a daily basis by a physician. If delirium was present or suspected, a geriatrician was consulted and the diagnosis was confirmed based on the DSM-IV criteria.

From 2015 onward, a geriatrician was also consulted prior to surgery as standard care for each patient with a hip fracture.

\section{Adverse events and clinical outcome}

The secondary objective of this study was to examine the effect of delirium on a number of complications (medical and surgical adverse events), the length of stay in hospital, mortality (30 days, 3 months, 1 year) and the number of patients newly discharged to a nursing home.

\section{Statistical analysis}

Categorical variables are presented as frequencies and percentages. Continuous variables are presented as mean $( \pm \mathrm{SD})$. The nature of the correlation (eg, linear and quadratic) between candidate predictors and outcome was graphically assessed by locally weighted scatterplot smoothing curves. For a nonlinear correlation, cut-off points were determined based on the observed distribution, clinical grounds, or literature. Associations between potential predictors and the primary outcome delirium were tested by univariable logistic regression. Significant associations observed in univariable analysis and significant variables reported in the literature were included in the multivariable model analyzing the association with delirium. ${ }^{3}$ Subsequently, backward elimination was performed until all remaining variables reached a $P$-value $<0.10$, controlling for the NHFS score. ${ }^{21}$ The mortality risk after delirium was analyzed with a multivariable model including significant variables reported in literature. ${ }^{12,22}$ Statistical analyses were performed using Stata version 14.0 (StataCorp, College Station, TX, USA), and all statistical tests were two-sided with a significance level of $P<0.05$. Mortality rates were estimated and displayed using the Kaplan-Meier estimator (Figure 2).

\section{Results \\ Baseline characteristics}

Table 1 shows the characteristics, including sub-stratification according to the delirium status, of all 463 patients in the present study. The mean age of the patients was $81 \pm 8$ years, $67 \%(n=310)$ were female, and almost $50 \%(n=225)$ of the patients had an ASA score of $\geq 3$. A total of $131(30 \%)$ patients were admitted to a semi-independent or nursing home facility. According to the DOSS and CAM scores, combined with geriatric assessment, 121 patients (26\%) had a delirium during hospital stay. The mean age in the delirium group was significantly higher ( 84 vs 80 years, $P<0.001$ ), and more patients in the delirium group ( $41 \%$ vs $16 \%$ ) had an underlying form of dementia before admission $(P<0.001)$. Significantly more patients were admitted to a semi-independent nursing home $(\mathrm{n}=53, P=0.003)$ and nursing home $(\mathrm{n}=78, P=0.018)$. Median duration of a delirium during admission was 5 days (IQR 3-7).

\section{Predictors of postoperative delirium}

The results of the univariable analysis of the risk and prognostic factors are displayed in Table 2. The results suggest the risk of delirium is increased by age (OR 1.07, [95\% CI 1.04-1.11], $P<0.001$ ), NHFS (OR 1.65, [95\% CI $1.38-1.97$ ],$P<0.001$ ), ASA score 3 and 4 (OR 1.61, [95\% CI 1.05-2.46], $P=0.028$ ), previous dementia (OR 3.84, [95\% CI 2.40-6.16], $P<0.001$ ), glomerular filtration rate (GFR) on admission (OR 0.99, [95\% CI 0.98-0.99], $P=0.028$ ), semiindependent nursing home (OR 2.53, [95\% CI 1.37-4.67], $P=0.003$ ) or nursing home (OR 1.92, [95\% CI 1.12-3.31], $P=0.018$ ), and an infection during admission (pneumonia, deep surgical site infection, or urinary tract infection) (OR 1.91, [95\% CI 1.21-3.01], $P=0.005$ ). No significant higher risk for delirium was found for gender, BMI, blood loss, type of anesthesia, reoperation rate, time to surgery, and surgery performed around daily working hours. 


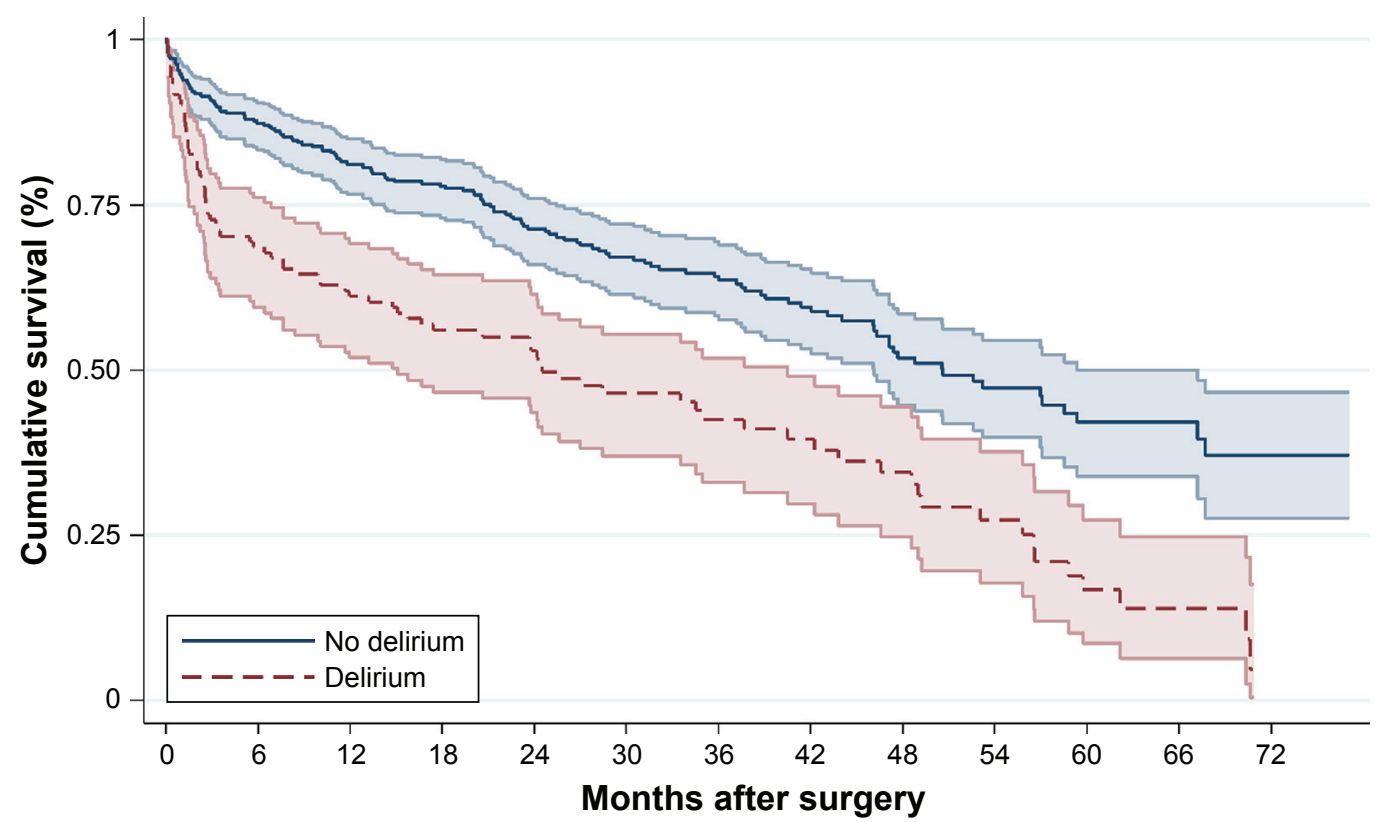

Figure 2 Mortality rates in patients with and without delirium during admission (Kaplan-Meier curve).

Table I Baseline characteristics of 463 included patients

\begin{tabular}{|c|c|c|c|}
\hline \multirow[t]{2}{*}{ Variable } & Overall (463) & Delirium (12I) & No delirium (342) \\
\hline & n/known n (\%) & n/known n (\%) & n/known n (\%) \\
\hline Age, years (mean $\pm S D$ ) & $81 \pm 8$ & $84 \pm 8$ & $80 \pm 8$ \\
\hline \multicolumn{4}{|l|}{ Gender } \\
\hline Female & $310 / 463(67)$ & $8 I / 12 I(67)$ & $229 / 342(67)$ \\
\hline Male & $153 / 463(33)$ & $40 / 121(33)$ & $113 / 342(33)$ \\
\hline \multicolumn{4}{|l|}{ ASA score } \\
\hline 1 & $7 / 456(2)$ & $1 / 117(1)$ & 6/339(2) \\
\hline 2 & $224 / 456(49)$ & $48 / 117(4 I)$ & $176 / 339(52)$ \\
\hline 3 & $214 / 456(47)$ & $66 / 117(56)$ & I48/339 (43) \\
\hline 4 & $1 \mathrm{I} / 456(2)$ & $2 / 117(2)$ & $9 / 339(3)$ \\
\hline NHFS (median, IQR) & $5(4-5)$ & $5(4-6)$ & $4(3-5)$ \\
\hline \multicolumn{4}{|l|}{ Medical history } \\
\hline Dementia & $104 / 463(23)$ & $49 /|2|(4 I)$ & $55 / 342(16)$ \\
\hline Diabetes mellitus & $91 / 463(20)$ & $3 I / I 2 I(26)$ & $60 / 342(18)$ \\
\hline Obesity (BMI >30 kg/m²) & $48 / 404(12)$ & $11 / 99(11)$ & $37 / 305(12)$ \\
\hline Underweight (BMI $\left.<18.5 \mathrm{~kg} / \mathrm{m}^{2}\right)$ & $27 / 404(7)$ & $10 / 99(10)$ & $17 / 305(6)$ \\
\hline GFR (glomerular filtration rate) on admission (median, IQR) & $68(50-85)$ & $64(45-80)$ & $70(52-86)$ \\
\hline Parkinson & $22 / 463(5)$ & $7 / 121(6)$ & $15 / 342(4)$ \\
\hline COPD & $50 / 463(1 \mathrm{I})$ & $13 / 12 \mid(1 \mid)$ & $37 / 342(I I)$ \\
\hline TIA & $57 / 463(12)$ & $18 / 121(15)$ & $39 / 342(11)$ \\
\hline Cerebrovascular accident & $42 / 463(9)$ & $13 / 12 \mid(11)$ & $29 / 342(8)$ \\
\hline Cardiovascular disease & $283 / 463(6 I)$ & $79 / 121(65)$ & $204 / 342(60)$ \\
\hline Myocardial infarction & $50 / 463(\mathrm{II})$ & $14 /|2|(12)$ & $36 / 342(I I)$ \\
\hline Previous hip fracture surgery & $45 / 462(10)$ & $15 / 120(13)$ & $30 / 342(9)$ \\
\hline Hemoglobin on admission (mmol/L) & $8(7.4-8.6)$ & $8.1(7.3-8.6)$ & $8(7.4-8.6)$ \\
\hline Malignancy in $<20$ years & $72 / 463(16)$ & $18 / 12 \mid(15)$ & $54 / 342(16)$ \\
\hline \multicolumn{4}{|l|}{ Residential status } \\
\hline Home & $320 / 45 I(7 I)$ & $66 / 113(58)$ & $254 / 338(75)$ \\
\hline Semi-independent nursing home & $53 / 451(12)$ & $21 / 113(19)$ & $32 / 338(10)$ \\
\hline Nursing home & $78 / 45 \mid(17)$ & $26 / 113(23)$ & $52 / 338(15)$ \\
\hline
\end{tabular}


Table I (Continued)

\begin{tabular}{|c|c|c|c|}
\hline \multirow[t]{2}{*}{ Variable } & Overall (463) & Delirium (12I) & No delirium (342) \\
\hline & n/known n (\%) & n/known n (\%) & n/known n (\%) \\
\hline \multicolumn{4}{|l|}{ Walking aids } \\
\hline None & $269 / 420(64)$ & $59 / 105(56)$ & $210 / 315(67)$ \\
\hline Walking cane & $8 / 420(2)$ & $5 / 105(5)$ & $3 / 315(1)$ \\
\hline Rollator & $122 / 420(29)$ & $37 / 105(35)$ & $85 / 315(27)$ \\
\hline Wheelchair or mobility scooter & $21 / 420(5)$ & $4 / 105(4)$ & $17 / 315(5)$ \\
\hline Polypharmacy (>4 medications) & $245 / 463(53)$ & $69 / 121(57)$ & $176 / 342(5 \mid)$ \\
\hline \multicolumn{4}{|l|}{ SNAQ-RC } \\
\hline 0 & $324 / 382(85)$ & $83 / 106(78)$ & $24 I / 276(87)$ \\
\hline $\mathrm{I}-2$ & $33 / 382(9)$ & $14 / 106(13)$ & $19 / 276(7)$ \\
\hline 3 & $25 / 382(7)$ & $9 / 106(8)$ & $16 / 276(6)$ \\
\hline Katz-ADL, (0-I0), (median, IQR) & $5(4-6)$ & $5(4-6)$ & $5(4-6)$ \\
\hline
\end{tabular}

Abbreviations: ASA, American Society of Anesthesiology; BMI, body mass index; Katz-ADL, Katz Index of Independence in Activities of Daily Living; SNAQ-RC, Short Nutritional Assessment Questionnaire for Residential Care; TIA, transient ischemic attack; NHFS, Nottingham hip fracture score.

Table 2 Univariable analyses of risk and prognostic factors ${ }^{\mathrm{a}}$

\begin{tabular}{|c|c|c|c|c|c|}
\hline \multirow[t]{2}{*}{ Variable } & All patients (463) & Delirium (12I) & No delirium (342) & \multirow[t]{2}{*}{ OR $(95 \% \mathrm{Cl})$} & \multirow[t]{2}{*}{$P$-value } \\
\hline & n/known n & n/known n (\%) & n/known n (\%) & & \\
\hline Age, years (mean $\pm S D$ ) & $8 I \pm 8$ & $84 \pm 8$ & $80 \pm 8$ & $1.07(1.04-1.11)$ & $<\mathbf{0 . 0 0 I ^ { b }}$ \\
\hline Female gender & $310 / 463(67)$ & $8 I / I 2 I(67)$ & $229 / 342(67)$ & $0.99(0.64-1.55)$ & 0.997 \\
\hline NHFS (median \pm SD) & $5(4-5)$ & $5(4-6)$ & $4(3-5)$ & $1.65(1.38-197)$ & $<0.00 \mathrm{I}^{\mathrm{C}}$ \\
\hline \multicolumn{6}{|l|}{ ASA score } \\
\hline I and 2 (reference) & $23 I / 456(5 I)$ & $49 / 117(42)$ & $182 / 339(54)$ & - & - \\
\hline 3 and 4 & $225 / 456(49)$ & $68 / 117(58)$ & I57/339(46) & $1.61(1.05-2.46)$ & 0.028 \\
\hline \multicolumn{6}{|l|}{ Medical history } \\
\hline Dementia & $104 / 463(23)$ & $49 / I 2 I(4 I)$ & $55 / 342(16)$ & $3.84(2.40-6.16)$ & $<0.001$ \\
\hline Diabetes mellitus & $91 / 463(20)$ & $3|/| 2 \mid(26)$ & $60 / 342(18)$ & $1.62(0.99-2.65)$ & 0.056 \\
\hline GFR on admission (median, IQR) & $68(50-85)$ & $64(45-80)$ & $70(52-86)$ & $0.99(0.98-0.99)$ & 0.028 \\
\hline Parkinson & $22 / 463(5)$ & $7 / 121(6)$ & I5/342(4) & $1.34(0.53-3.37)$ & 0.532 \\
\hline COPD & $50 / 463(I I)$ & $|3 /| 2 \mid(||)$ & $37 / 342(11)$ & $0.99(0.5 I-1.94)$ & 0.982 \\
\hline TIA & $57 / 463(12)$ & $|8 / I 2|(15)$ & $39 / 342(11)$ & $1.40(0.76-2.57)$ & 0.277 \\
\hline Cerebrovascular accident & $42 / 463(9)$ & $|3 /| 2 \mid(\mid I)$ & $29 / 342(8)$ & $1.36(0.68-2.73)$ & 0.387 \\
\hline Cardiovascular disease & $283 / 463(6 I)$ & $79 / 121(65)$ & $204 / 342(60)$ & $1.27(0.83-1.96)$ & 0.275 \\
\hline Myocardial infarction & $50 / 463(11)$ & $|4 /| 2 \mid(\mid 2)$ & $36 / 342(11)$ & I.II (0.58-2.14) & 0.751 \\
\hline Previous hip fracture surgery & $45 / 462(10)$ & $15 / 120(13)$ & $30 / 342(9)$ & $1.49(0.77-2.87)$ & 0.238 \\
\hline Hemoglobin on admission (mmol/L) & $8(7.4-8.6)$ & $8.1(7.3-8.6)$ & $8(7.4-8.6)$ & $1.03(0.83-1.29)$ & 0.774 \\
\hline Malignancy in $<20$ years & $72 / 463(16)$ & $|8 / I 2|(15)$ & $54 / 342(16)$ & $0.93(0.52-1.66)$ & 0.812 \\
\hline Polypharmacy ( $>4$ medications) & $245 / 463(53)$ & $69 / 121(57)$ & $176 / 342(5 \mathrm{I})$ & $1.25(0.82-1.90)$ & 0.292 \\
\hline \multicolumn{6}{|l|}{ Residential status } \\
\hline Home (reference) & $320 / 45 I(7 I)$ & $66 / 113(58)$ & $254 / 338(75)$ & - & - \\
\hline Semi-independent nursing home & $53 / 45 I(12)$ & $21 / 113(19)$ & $32 / 338(10)$ & $2.53(1.37-4.67)$ & 0.003 \\
\hline Nursing home & $78 / 45 \mid(17)$ & $26 / 113(23)$ & $52 / 338(15)$ & $1.92(1.12-3.31)$ & 0.018 \\
\hline \multicolumn{6}{|l|}{ Walking aids } \\
\hline None (reference) & $277 / 420(66)$ & $64 / 105(6 I)$ & $213 / 315(68)$ & - & - \\
\hline Rollator & $122 / 420(29)$ & $37 / 105(35)$ & $85 / 315(27)$ & $1.45(0.90-2.33)$ & 0.127 \\
\hline Wheelchair/mobility scooter & $21 / 420(5)$ & $4 / 105(4)$ & $17 / 315(5)$ & $0.78(0.25-2.4 \mathrm{I})$ & 0.670 \\
\hline
\end{tabular}

(Continued) 
Table 2 (Continued)

\begin{tabular}{|c|c|c|c|c|c|}
\hline \multirow[t]{2}{*}{ Variable } & \multirow{2}{*}{\begin{tabular}{|l|} 
All patients (463) \\
n/known $n$
\end{tabular}} & \multirow{2}{*}{$\begin{array}{l}\text { Delirium (12I) } \\
\text { n/known n (\%) }\end{array}$} & \multirow{2}{*}{\begin{tabular}{|l|} 
No delirium (342) \\
n/known n (\%) \\
\end{tabular}} & \multirow[t]{2}{*}{ OR $(95 \% \mathrm{Cl})$} & \multirow[t]{2}{*}{$P$-value } \\
\hline & & & & & \\
\hline \multicolumn{6}{|l|}{ Time to surgery } \\
\hline$\leq 24$ hours & $|23 / 46|(27)$ & $36 / 121(30)$ & $87 / 340(26)$ & $1.23(0.78-1.95)$ & 0.374 \\
\hline$\geq 24$ hours (reference) & $338 / 46 \mid(73)$ & $85 / I 21(70)$ & $253 / 340(74)$ & - & - \\
\hline \multicolumn{6}{|l|}{ Moment of the week } \\
\hline During the week (reference) & $348 / 463(75)$ & $83 / 121(69)$ & $265 / 342(77)$ & - & - \\
\hline Weekend & II5/463 (25) & $38 /|2|(3 \mid)$ & $77 / 342(23)$ & $1.57(0.99-2.50)$ & 0.053 \\
\hline \multicolumn{6}{|l|}{ Moment of the day } \\
\hline Daytime (reference) & $403 / 463(87)$ & $104 / 121(86)$ & 299/342 (87) & - & - \\
\hline Night time (18:00-08:00) & $60 / 463(13)$ & $17 / 121(14)$ & $43 / 342(13)$ & $1.13(0.62-2.08)$ & 0.678 \\
\hline \multicolumn{6}{|l|}{ Anesthesia } \\
\hline Spinal (reference) & $356 / 463(77)$ & $89 / 121(74)$ & $267 / 342(78)$ & - & - \\
\hline General & $107 / 463(23)$ & $32 / 121(26)$ & $75 / 342(22)$ & $1.28(0.79-2.07)$ & 0.312 \\
\hline Reoperation & $23 / 455(5)$ & $6 / 119(5)$ & $17 / 336(5)$ & $0.99(0.38-2.59)$ & 0.994 \\
\hline \multicolumn{6}{|l|}{ Surgery time } \\
\hline$\leq 45$ minutes & $45 / 462(10)$ & $|5 /| 2 \mid(\mid 2)$ & $30 / 341(9)$ & $1.49(0.77-2.89)$ & 0.238 \\
\hline 45-90 minutes (reference) & $378 / 462(82)$ & $95 / 121(79)$ & $283 / 34 \mid(83)$ & - & - \\
\hline$\geq 90$ minutes & $39 / 462(8)$ & $|I / / 2|(9)$ & $28 / 34 \mid(8)$ & $1.17(0.56-2.44)$ & 0.675 \\
\hline \multicolumn{6}{|l|}{ SNAQ } \\
\hline 0 & $324 / 382(85)$ & $83 / 106(78)$ & $24 I / 276(87)$ & - & - \\
\hline $1-2$ & $33 / 382(9)$ & $14 / 106(13)$ & 19/276 (7) & $2.14(1.03-4.46)$ & 0.042 \\
\hline$\geq 3$ & $25 / 382(7)$ & $9 / 106(8)$ & $16 / 276(6)$ & $1.63(0.70-3.84)$ & 0.260 \\
\hline Infection during admission & $|I 6 / 46|(25)$ & $42 / 121(35)$ & $74 / 340(22)$ & $1.91(1.21-3.01)$ & 0.005 \\
\hline Hemoglobin loss (mmol/L), median \pm SD & $1.4(I-2.1)$ & $1.4(I-2.1)$ & $1.4(0.9-2)$ & $0.93(0.72-1.20)$ & 0.598 \\
\hline \multicolumn{6}{|l|}{ BMI $\left(\mathrm{kg} / \mathrm{m}^{2}\right)$} \\
\hline$\leq 18.5$ & $27 / 404(7)$ & $10 / 99(10)$ & $17 / 305(6)$ & $1.89(0.83-4.30)$ & 0.128 \\
\hline 18.5-30.0 (reference) & $329 / 404(8 I)$ & $78 / 99$ (79) & $25 \mathrm{I} / 305(82)$ & - & - \\
\hline$\geq 30.0$ & $48 / 404(12)$ & II/99 (II) & $37 / 305$ (I2) & $0.96(0.47-1.96)$ & 0.904 \\
\hline
\end{tabular}

Notes: apearson's chi-squared test was used unless specified otherwise. 'btudent's t-test. 'Wilcoxon rank-sum test. Bold values indicate statistical significance.

Abbreviations: NHFS, Nottingham hip fracture score; ASA, American Society of Anesthesiology; BMI, body mass index; GFR, glomerular filtration rate; TIA, transient ischemic attack; SNAQ, Short Nutritional Assessment Questionnaire.

Significant associations observed in univariable analysis were included in a multivariable model, correcting for the NHFS (Table 3). The multivariable model showed that dementia (OR 2.75, [95\% CI 1.51-5.02], $P=0.001$ ), age (OR 1.05, [95\% CI 1.02-1.09], $P=0.005$ ), and an infection during admission (pneumonia, deep surgical site infection, or urinary tract infection) (OR 1.23, [95\% CI 1.00-1.50], $P=0.046)$ were significant independent prognostic factors for the development of a delirium, with the largest effect for dementia.

\section{Clinical outcome}

Clinical outcomes after delirium are shown in Table 4 . The median length of the primary in-hospital stay in the non-dementia group with a delirium was 11 days, which was not significant compared with 7 days in the nondelirium dementia group $(P=0.128)$. Moreover, patients with a delirium during admission were significantly more often discharged to nursing home (OR 7.06, [95\% CI 2.13-23.41], $P=0.001$ ) or semi-independent nursing home (OR 11.4, [95\% CI 3.4-38.1], $P<0.001$ ) facilities.

Table 3 Multivariate analyses of risk factors in delirium

\begin{tabular}{l|l|l|l}
\hline Factor & OR & $\mathbf{9 5 \%} \mathbf{C l}$ & $\boldsymbol{P}$-value \\
\hline Age, years & 1.05 & $1.02-1.09$ & $\mathbf{0 . 0 0 5}$ \\
NHFS & 1.19 & $0.93-1.52$ & 0.160 \\
Dementia & 2.75 & $1.51-5.02$ & $\mathbf{0 . 0 0 1}$ \\
Infection $^{\mathrm{a}}$ & 1.23 & $1.00-1.50$ & $\mathbf{0 . 0 4 6}$
\end{tabular}

Note: anfection: pneumonia, deep surgical site infection, or urinary tract infection during admission. Bold values indicate statistical significance.

Abbreviation: NHFS, Nottingham hip fracture score. 
Table 4 Clinical outcomes after delirium

\begin{tabular}{|c|c|c|c|c|c|}
\hline \multirow[t]{2}{*}{ Variable } & \multirow{2}{*}{\begin{tabular}{|l|} 
All patients (463) \\
n/known $n$ \\
\end{tabular}} & \multirow{2}{*}{$\begin{array}{l}\text { Delirium (1 2I) } \\
\text { n/known n (\%) } \\
\end{array}$} & \multirow{2}{*}{$\begin{array}{l}\text { No delirium (342) } \\
\text { n/known n (\%) }\end{array}$} & \multirow[t]{2}{*}{ OR (95\% CI) } & \multirow[t]{2}{*}{$P$-value } \\
\hline & & & & & \\
\hline Length of delirium (days) (median IQR) & - & $5(3-7)$ & - & & \\
\hline \multicolumn{6}{|l|}{ Length of hospital stay (median IQR) } \\
\hline Dementia & $5(4-9)$ & $6(4-10)$ & $5(4-7)$ & & 0.693 \\
\hline Non-dementia & $8(6-11)$ & II (7-14) & $7(6-10)$ & & 0.128 \\
\hline \multicolumn{6}{|l|}{ Discharge destination without dementia } \\
\hline New to nursing home & $211 / 282(75)$ & $48 / 50(96)$ & $163 / 232(70)$ & $7.06(2.13-23.41)$ & 0.001 \\
\hline Home (reference) & $73 / 336(22)$ & $3 / 62(5)$ & $70 / 274(26)$ & - & - \\
\hline Semi-independent nursing home & $152 / 336(45)$ & $50 / 62(81)$ & $102 / 274(37)$ & II.4 (3.4-38.I) & $<0.001$ \\
\hline Nursing home & $111 / 336(33)$ & $9 / 62(15)$ & $102 / 274(37)$ & $2.06(0.54-7.88)$ & 0.291 \\
\hline \multicolumn{6}{|l|}{ Discharge destination with dementia } \\
\hline New to nursing home & $18 / 24(75)$ & $11 / 13(85)$ & $7 / 11(64)$ & $2.09(0.36-12.32)$ & 0.413 \\
\hline Home (reference) & $7 / 92(1)$ & $3 / 43(I)$ & $4 / 49(I)$ & - & - \\
\hline Semi-independent nursing home & $79 / 92(86)$ & $37 / 43(86)$ & $42 / 49(86)$ & $1.17(0.25-5.59)$ & 0.840 \\
\hline Nursing home & 6/92 (I) & $3 / 43(1)$ & $3 / 49(1)$ & $1.33(0.15-11.93)$ & 0.797 \\
\hline \multicolumn{6}{|l|}{ Mortality } \\
\hline In hospital & $21 / 463(5)$ & $10 / 121(8)$ & $1 \mathrm{I} / 342(3)$ & $2.71(1.12-6.55)$ & 0.027 \\
\hline 30 days & $33 / 463(7)$ & $12 / I 2 I(10)$ & $21 / 342(6)$ & $1.68(0.80-3.53)$ & 0.169 \\
\hline I year & $113 / 463(24)$ & $47 / 121(39)$ & $66 / 342(19)$ & $2.65(1.68-4.18)$ & $<0.001$ \\
\hline
\end{tabular}

Note: Bold values indicate statistical significance.

The overall survival rate of the population is shown in Figure 2 (Kaplan-Meier analysis). The 30-day all-cause mortality was $7 \%(n=33)$ (delirium-positive $10 \%$ vs delirium-negative 6\%). After 1 year of follow-up, the allcause mortality was $24 \%(n=113)$ in all patients. In the delirium-positive group, 39\% $(\mathrm{n}=47)$ died within 1 year after surgery (OR 2.65, [95\% CI 1.68-4.18], $P<0.001$ ) compared with $19 \%(\mathrm{n}=66)$ in the non-delirium group. After correction for other confounding factors, delirium remained a significant predictor for 1-year mortality (Table 5). A total of 152 patients were followed up at the outpatient clinics for post-discharge control. The other patients were followed up by a nursing home physician at their residence or by a general practitioner at home.

Table 5 Multivariate analyses of risk factors for mortality

\begin{tabular}{l|l|l|l}
\hline Factor & OR & $\mathbf{9 5 \%} \mathbf{C l}$ & $\boldsymbol{P}$-value \\
\hline Delirium & 1.93 & $1.13-3.28$ & $\mathbf{0 . 0 1 6}$ \\
Age (years) & 0.98 & $0.95-1.01$ & 0.332 \\
NHFS & 1.60 & $1.25-2.06$ & $\mathbf{0 . 0 0 1}$ \\
Dementia & 1.27 & $0.69-2.34$ & 0.445 \\
Reoperation $^{\mathrm{a}}$ & 2.83 & $1.08-7.41$ & $\mathbf{0 . 0 3 4}$ \\
Infection $^{\mathrm{b}}$ & 1.35 & $0.78-2.40$ & 0.285 \\
\hline
\end{tabular}

Notes: aReoperation for a hematoma, luxation, or deep surgical site infection; binfection: pneumonia, deep surgical site infection, or urinary tract infection. Bold values indicate statistical significance.

Abbreviation: NHFS: Nottingham hip fracture score.

\section{Discussion}

Surgery in elderly patients has a high impact, especially in the frail population with a hip fracture. Efficient and multidisciplinary perioperative management with early treatment of complications is important to reduce postoperative mortality. The most frequent complication during admission after hip fracture surgery is delirium with incidence rates between $23 \%$ and $39 \% .^{6-8}$ In line with previous literature, the incidence rate of delirium in our population was $26 \%$ $(\mathrm{n}=121))^{6-8,23}$ The results of the multivariable analysis identified the following independent potential risk factors causing delirium: predisposing dementia, age and an infection during admission (pneumonia, deep surgical site infection, or urinary tract infection). GFR and the NHFS became insignificant factors after multivariate analysis.

\section{Perioperative risk factors}

In previous literature, cognitive impairment or dementia is one of the most important risk factors for delirium. ${ }^{16}$ Voyer et al reported the severity of dementia is a significant indicator for delirium severity, and higher morbidity and mortality rates have been described with delirium superimposed on dementia. ${ }^{24-26}$ The incidence of delirium in patients with dementia ranges between $57 \%$ and $62 \%$ during admission in previous literature. ${ }^{9,27}$ In this study, among patients who underwent hip fracture surgery, $41 \%(n=49)$ with dementia 
developed a delirium during admission, and indeed dementia was a significant factor for developing a delirium. Delirium could thus be a marker of the vulnerable brain with diminished reserve capacity as is present in patient with dementia. Accumulating evidence, however, suggests that delirium itself might lead to permanent cognitive decline and dementia in some patients. ${ }^{28}$

Age was a significant predictor for delirium, with a median age of 84 years in the delirium group and 80 years in the non-delirium group. This result is in line with reported previous literature among hip fracture patients, including Kalisvaart et al (603 hip surgery patients) and Lee et al (425 hip surgery patients) who found that age was an independent predictive factor for delirium. ${ }^{16,29}$ During admission, patients with delirium developed significantly more often an infection (pneumonia, urinary tract infection, or wound infection) compared to patients without a delirium ( $35 \%$ vs $22 \%$ ). Since the diagnosis of an infection was not done in all patients before the occurrence of a delirium, we cannot conclude that an infection is an independent prognostic factor for delirium. However, our study results suggest infections during admission are associated with the occurrence of a delirium during admission.

In accordance with previous literature on hip fracture surgery, our findings showed no significant correlation with time to surgery and the development of a delirium. Moreover, surgery during the weekend or around normal working hours did not show a significant difference. General anesthesia during surgery was performed in $25 \%$ of the patients, and there was no difference in delirium prevalence compared to spinal anesthesia as reported earlier. ${ }^{27,30}$ The correlation between a prolonged length of surgery and delirium has not been previously described, and in accordance we did not find a significant correlation between length of surgery and delirium. ${ }^{27}$

\section{Clinical outcomes}

In line with the literature, the overall survival rate in the delirium-positive group (39\%) was significantly lower after 1 year of follow-up than in the non-delirium group (19\%), even after correcting for other confounding risk factors as suggested by Dolan et al. ${ }^{12}$ After correction, also the NHFS and a reoperation after initial surgery remained a significant predictor for 1-year mortality.

The most effective method in delirium care is prevention of its occurrence. Several studies demonstrated that delirium is preventable in one-third of the cases by performing multicomponent interventions during admission. ${ }^{28}$ When high-risk patients are identified earlier, appropriate preventive arrangements can be implemented and most likely will result in a lower delirium rate. As a result, related clinical outcomes as hospital stay and mortality rates may improve significantly. In case of planned, major elective surgery in elderly, early identification of high-risk patients is most effective. Outpatient consultation of a multidisciplinary coordinated team of health care professionals can screen patients on frailty and risk for delirium. Instantly, the modifiable risk factors could be optimized. However, in hip fracture patients, surgery is never planned and by definition semi-acute. The options for preoperative optimization are therefore limited. Still, preoperative cognitive assessment may be one of the most useful methods of identifying those who are at high risk for postoperative delirium and is of major importance to optimize delirium care.

\section{Strengths and limitations}

Baseline characteristics and clinical data had to be completed retrospectively, leading to a potential absence of data on confounding factors. Yet, the prospective hip fracture database prevents selection bias and absence of data regarding most variables. Cognitive impairment or dementia was evaluated using medical notes and no distinction was made between the severities of cognitive impairment. In addition, dementia often presents itself during admission, which might result in an underestimation of the number of patients with cognitive disorders in this study.

\section{Conclusion}

Our findings showed that, of all potential risk factors suggested in previous literature, only dementia, age, and an infection during admission were significantly associated with an increased risk of delirium after hip fracture surgery. In turn, delirium was found to result in increased admission to nursing homes and a higher risk of mortality after 1 year.

\section{Disclosure}

The authors report no conflicts of interest in this work.

\section{References}

1. Leon DA. Trends in European life expectancy: A salutary view. Int $J$ Epidemiol. 2011;40(2):271-277.

2. Kanis JA, Odén A, Mccloskey EV, et al. A systematic review of hip fracture incidence and probability of fracture worldwide. Osteoporos Int. 2012;23(9):2239-2256.

3. Jameson SS, Khan SK, Baker P, et al. A national analysis of complications following hemiarthroplasty for hip fracture in older patients. QJM. 2012;105(5):455-460.

4. Nichols CI, Vose JG, Nunley RM. Clinical outcomes and 90-day costs following Hemiarthroplasty or total hip arthroplasty for hip fracture. J Arthroplasty. 2017;32(9S):S128-S134. 
5. de Jong L, Klem T, Kuijper TM, Roukema GR. Factors affecting the rate of surgical site infection in patients after hemiarthroplasty of the hip following a fracture of the neck of the femur. Bone Joint J. 2017;99-B(8): 1088-1094.

6. Krogseth M, Wyller TB, Engedal K, Juliebø V. Delirium is a risk factor for institutionalization and functional decline in older hip fracture patients. J Psychosom Res. 2014;76(1):68-74.

7. Gleason LJ, Schmitt EM, Kosar CM, et al. Effect of delirium and other major complications on outcomes after elective surgery in older adults JAMA Surg. 2015;150(12):1134-1140.

8. Yang Y, Zhao X, Dong T, Yang Z, Zhang Q, Zhang Y. Risk factors for postoperative delirium following hip fracture repair in elderly patients: a systematic review and meta-analysis. Aging Clin Exp Res. 2017; 29(2):115-126.

9. Witlox J, Eurelings LS, de Jonghe JF, Kalisvaart KJ, Eikelenboom P, van Gool WA. Delirium in elderly patients and the risk of postdischarge mortality, institutionalization, and dementia: a meta-analysis. JAMA. 2010;304(4):443-451.

10. Krogseth M, Watne LO, Juliebø V, et al. Delirium is a risk factor for further cognitive decline in cognitively impaired hip fracture patients. Arch Gerontol Geriatr. 2016;64:38-44.

11. Bellelli G, Mazzola P, Morandi A, et al. Duration of postoperative delirium is an independent predictor of 6-month mortality in older adults after hip fracture. J Am Geriatr Soc. 2014;62(7):1335-1340.

12. Dolan MM, Hawkes WG, Zimmerman SI, et al. Delirium on hospital admission in aged hip fracture patients: prediction of mortality and 2-year functional outcomes. J Gerontol A Biol Sci Med Sci. 2000;55(9): M527-M534.

13. Marcantonio ER, Flacker JM, Wright RJ, Resnick NM. Reducing delirium after hip fracture: a randomized trial. J Am Geriatr Soc. 2001; 49(5):516-522.

14. Zhang Y, Shan GJ, Zhang YX, et al. Propofol compared with sevoflurane general anaesthesia is associated with decreased delayed neurocognitive recovery in older adults. Br J Anaesth. 2018;121(3):595-604.

15. Su X, Meng ZT, Wu XH, et al. Dexmedetomidine for prevention of delirium in elderly patients after non-cardiac surgery: a randomised, doubleblind, placebo-controlled trial. Lancet. 2016;388(10054):1893-1902.

16. Kalisvaart KJ, Vreeswijk R, de Jonghe JF, van der Ploeg T, van Gool WA, Eikelenboom P. Risk factors and prediction of postoperative delirium in elderly hip-surgery patients: implementation and validation of a medical risk factor model. J Am Geriatr Soc. 2006;54(5):817-822.

17. Oh ES, Li M, Fafowora TM, et al. Preoperative risk factors for postoperative delirium following hip fracture repair: a systematic review. Int J Geriatr Psychiatry. 2015;30(9):900-910.
18. Choi YH, Kim DH, Kim TY, Lim TW, Kim SW, Yoo JH. Early postoperative delirium after hemiarthroplasty in elderly patients aged over 70 years with displaced femoral neck fracture. Clin Interv Aging. 2017; 12:1835-1842.

19. Maxwell MJ, Moran CG, Moppett IK. Development and validation of a preoperative scoring system to predict 30 day mortality in patients undergoing hip fracture surgery. Br J Anaesth. 2008;101(4):511-517.

20. Moppett IK, Parker M, Griffiths R, Bowers T, White SM, Moran CG. Nottingham hip fracture score: longitudinal and multi-assessment. Br J Anaesth. 2012;109(4):546-550.

21. White IR, Royston P, Wood AM. Multiple imputation using chained equations: issues and guidance for practice. Stat Med. 2011;30(4): 377-399.

22. Kat MG, de Jonghe JF, Vreeswijk R, et al. Mortality associated with delirium after hip-surgery: a 2-year follow-up study. Age Ageing. 2011; 40(3):312-318.

23. Fick DM, Steis MR, Waller JL, Inouye SK. Delirium superimposed on dementia is associated with prolonged length of stay and poor outcomes in hospitalized older adults. J Hosp Med. 2013;8(9):500-505.

24. Voyer P, Mccusker J, Cole MG, St-Jacques S, Khomenko L. Factors associated with delirium severity among older patients. J Clin Nurs. 2007; 16(5):819-831.

25. Fick D, Foreman M. Consequences of not recognizing delirium superimposed on dementia in hospitalized elderly individuals. J Gerontol Nurs. 2000;26(1):30-40.

26. Bellelli G, Frisoni GB, Turco R, Lucchi E, Magnifico F, Trabucchi M. Delirium superimposed on dementia predicts 12-month survival in elderly patients discharged from a postacute rehabilitation facility. J Gerontol A Biol Sci Med Sci. 2007;62(11):1306-1309.

27. Mosk CA, Mus M, Vroemen JP, et al. Dementia and delirium, the outcomes in elderly hip fracture patients. Clin Interv Aging. 2017;12:421-430.

28. Inouye SK, Westendorp RG, Saczynski JS. Delirium in elderly people. Lancet. 2014;383(9920):911-922.

29. Lee HB, Mears SC, Rosenberg PB, Leoutsakos JM, Gottschalk A, Sieber FE. Predisposing factors for postoperative delirium after hip fracture repair in individuals with and without dementia. J Am Geriatr Soc. 2011;59(12):2306-2313.

30. Ilango S, Pulle RC, Bell J, Kuys SS. General versus spinal anaesthesia and postoperative delirium in an orthogeriatric population. Australas J Ageing. 2016;35(1):42-47.
Clinical Interventions in Aging

\section{Publish your work in this journal}

Clinical Interventions in Aging is an international, peer-reviewed journal focusing on evidence-based reports on the value or lack thereof of treatments intended to prevent or delay the onset of maladaptive correlates of aging in human beings. This journal is indexed on PubMed Central, MedLine,

\section{Dovepress}

CAS, Scopus and the Elsevier Bibliographic databases. The manuscript management system is completely online and includes a very quick and fair peer-review system, which is all easy to use. Visit http://www.dovepress. com/testimonials.php to read real quotes from published authors. 